\title{
La indagación empírica en torno a la violencia en las escuelas mexicanas desde las voces del alumnado: Puntos críticos
}

\section{The Empirical Research on Violence in Mexican Schools from the Voices of Students: Critical Points}

\author{
Ursula Zurita Rivera \\ Facultad Latinoamericana de Ciencias Sociales, Ciudad de México, México \\ ORCID: https://orcid.org/0000-0002-7381-3550
}

Recibido 07-01-19 Revisado 11-01-19 Aprobado 13-03-19 En línea 14-03-19

\section{Correspondencia}

Email: uzurita@flacso.edu.mx

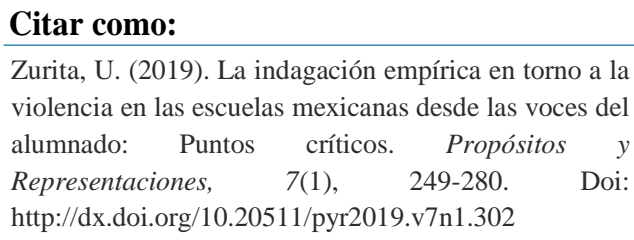

(C) Universidad San Ignacio de Loyola, Vicerrectorado de Investigación, 2019 


\section{Resumen}

La creciente visibilidad de la violencia en las escuelas ha propiciado múltiples demandas por conocer su estado en nuestras sociedades. Ante esto, son evidentes los retos conceptuales, analíticos y metodológicos enfrentados entre aquellos ocupados en su indagación empírica. Sin embargo, buena parte de quienes responden a esos desafíos, no analizan los procesos paralelos que se configuran conforme avanza la investigación. Este es el caso de los cambios acerca del papel de las y los alumnos en este esfuerzo colectivo. El objetivo de este documento es analizar los avances y dilemas de la indagación empírica de la violencia escolar en México, concentrándose en las implicaciones derivadas de la legitimación de las voces de niñas, niños y adolescentes (NNyA). Este examen basado en la literatura especializada sobre la violencia escolar y las voces del estudiantado, advierte que aunque se han generalizado los estudios que conciben a estas poblaciones como individuos capaces de proveer datos de dicho fenómeno mediante la aplicación de numerosas técnicas de investigación, persiste la idea de asumirlos como fuentes de información y como destinatarios pasivos y subordinados de las decisiones y acciones tomadas por los adultos. Este análisis se dirige a expertos, autoridades y líderes sociales que, al considerar al alumnado como interlocutores en la indagación empírica de la violencia escolar, están comprometidos con el reconocimiento del derecho a la participación de NNyA y del impulso de su ejercicio efectivo en las escuelas.

Palabras clave: Indagación empírica; voces de niñas, niños y adolescentes; violencia escolar; México.

\section{Summary}

The growing visibility of violence in schools has caused multiple demands to know their condition in our societies. Given this, the conceptual, analytical and methodological challenges faced between those engaged in their empirical research are evident. However, many of those who face these challenges do not analyze the parallel processes that are configured as the research progresses. This is the case of changes about the role of students in this collective effort. The objective of this document is to analyze the advances and dilemmas of the empirical research on violence in Mexico, focusing on the implications derived from the legitimization of the voices of children and adolescents. This analysis based on the specialized literature on school violence and the voices of students, warns that although studies that conceive these populations as individuals capable of providing data on this phenomenon through the application of numerous research techniques have been generalized, the idea of assuming them as sources of information and as passive and subordinated recipients of decisions and actions taken by adults. This analysis is aimed at experts, authorities and social leaders who, when considering the students as interlocutors in the empirical research on school violence, are committed to the recognition of the right to participation of children and adolescents, and the promotion of its effective exercise in schools.

Keywords: Empirical Research; Voices of Children and Adolescents; School Violence, Mexico.

\section{Introducción}

La creciente visibilidad del complejo y multidimensional fenómeno de la violencia en las escuelas, ha propiciado múltiples demandas para conocer su situación en nuestras sociedades. Ante estas exigencias, son evidentes los numerosos retos conceptuales, analíticos y metodológicos por todos aquellos interesados en su indagación empírica (Brown \& Munn, 2008; Henry \& Bracy, 2012; Gómez \& Zurita, 2013; Green, Felix, Sharkey, Furlong \& Kras, 2013; Hymel \& Swearer, 2015; Zych, Ortega-Ruiz, Del Rey, 2015; Scheithauera, Smith \& Samara, 2016). Sin embargo, poco se reflexiona en este contexto sobre el papel que tienen las y los alumnos en dicho esfuerzo colectivo. Por tal razón, en este documento se analizan los avances y desafíos alcanzados recientemente en México respecto a la indagación empírica de la violencia 
escolar, pero desde las voces de niñas, niños y adolescentes (NNyA). Al emprender este examen, se advierte que, si bien se han ampliado y en cierto modo, generalizado las iniciativas por considerar a estos integrantes de las comunidades escolares, como sujetos capaces de proveer información en la materia, todavía persiste la idea de asumirlos únicamente como fuentes de datos y como destinatarios pasivos de las decisiones y acciones que una amplia gama de actores, toman por ellos. Entre esos actores, además de los docentes y directores, se hallan las autoridades educativas y de otras áreas gubernamentales (como salud, desarrollo social, derechos humanos, por ejemplo), los especialistas, los expertos de las organizaciones civiles de distinta escala que intervienen en las decisiones y acciones para prevenir, atender y eliminar la violencia escolar. Por el contenido de este documento, este texto busca establecer un diálogo con quienes en la academia, el gobierno y los organismos civiles están, por un lado, a favor de considerar a las y los estudiantes como interlocutores en la indagación empírica de la violencia escolar; y, por otro, a defender el derecho a la participación de NNyA y de su ejercicio pleno en los espacios escolares.

Así, este trabajo busca abrir una ruta hasta ahora poco transitada para examinar los retos que acompañan a esta indagación empírica. Sin duda, hay ciertos avances relativos a la producción de marcos conceptuales, analíticos, metodológicos y técnicos para recabar y analizar las experiencias, opiniones y valoraciones que tienen las y los alumnos acerca de la violencia escolar, pero todavía es poco común que estos miembros de las comunidades escolares tomen un papel más activo y propositivo, en sintonía con los instrumentos legales existentes en México que establece el derecho a la participación según se establece en la Ley General de Derechos de Niñas, Niños y Adolescentes (Fielding, 2007, 2011; Mitra \& Serriere, 2012; Mockler \& GroundwaterSmith, 2015; Zurita, 2016). De hecho, si bien en los últimos años, se ha configurado un proceso de legitimación de las voces del alumnado en la gran mayoría de los procesos concernientes a la indagación empírica de la violencia escolar; esto no implica que NNyA intervengan en la formulación de iniciativas escolares y educativas para su atención, prevención y eliminación de las escuelas mexicanas (Zurita, 2013a; 2013b; 2018).

Por estas razones, aquí interesa analizar cuáles son esos avances y cuáles los desafíos de la indagación empírica en términos de la participación de NNyA en los procesos investigativos sobre la violencia escolar. En atención a este propósito central, el texto está organizado en cuatro partes. La primera hace un recuento breve de algunos de los retos vinculados con la indagación empírica de la violencia escolar. Quienes se han dedicado a esta tarea en la academia, el gobierno o las organizaciones civiles, saben que estos retos no reconocen fronteras de ningún tipo puesto que son inherentes al objeto de nuestro interés (Brown \& Munn, 2008, Henry \& Bracy, 2012; Hymel, McClure, Miller, Shumka \& Trach, 2015; Ortega-Ruiz, Del Rey, 2015; Hymel \& Swearer, 2015; Scheithauera, Smith \& Samara, 2016). En la siguiente parte se exponen algunos planteamientos de las Voces de las y los estudiantes concernientes al modelo de interacción entre NNyA y los docentes, directores y otros adultos en el marco de la producción de conocimiento, propuesto por Michel Fielding (2011). Estos planteamientos servirán para identificar, en la tercera parte, lo que se ha avanzado y también para reflexionar acerca de lo que falta por hacerse. Por último, se presentan algunos comentarios finales para continuar este debate.

Antes de desarrollar el trabajo, conviene señalar que la elaboración de este documento está sustentada en la revisión y uso, por una parte, de la literatura especializada en torno a la violencia escolar (Brown \& Munn, 2008, Henry \& Bracy, 2012; Gómez y Zurita, 2013; Hymel, McClure, Miller, Shumka \& Trach, 2015; Ortega-Ruiz, Del Rey, 2015; Hymel \& Swearer, 2015; Scheithauera, Smith \& Samara, 2016; Zurita, 2013, 2016); y, por otra, de la literatura de las Voces del alumnado (Tisdall, Kay \& Davis, 2004; Noyes, 2005; MackBeth, 2006; Fielding, 2007, 2011; Lundy, 2007; Mitra \& Serriere, 2012; Mockler \& Groundwater-Smith, 2015). Es importante decir que ambos acervos han sido utilizados en diversas investigaciones empíricas y documentales, individuales y colectivas, institucionales e interinstitucionales, realizadas desde hace más de una década de la participación social y la violencia en las escuelas de educación básica en México (Gómez \& Zurita, 2013; Zurita, 2010; 2013a; 2013b; 2016; 2018). 


\section{La indagación empírica sobre la violencia escolar en México.}

La información disponible sobre la violencia en las escuelas mexicanas muestra varios rasgos que se han identificado en otros países respecto a la emergencia y agudización de este problema (Brown \& Munn, 2008; Monks, Smith, Naylor, Barter, Ireland \& Coyne, 2009; Maunder \& Crafter, 2018); el cual si bien no es nuevo, si asumió rasgos inéditos a partir delos años noventa (Gómez \& Zurita, 2013; Hymel \& Swearer, 2015). Se sabe que es un fenómeno con características que lo asemejan a otros tipos de expresiones violentas -como la diferencia en cuanto al poder entre los individuos involucrados, el propósito de cometer algún tipo de daño, la combinación de factores individuales, escolares y sociales que propician su producción y reproducción, entre otros- pero en el espacio escolar la violencia asume cualidades, configuraciones, alcances e implicaciones únicas (Hymel \& Seawer, 2015; Smith, 2016; Zurita, 2016). En este panorama, ha habido notorios esfuerzos por establecer teorías, marcos analíticos y propuestas metodológicas para su estudio (Brown \& Munn, 2008; Gómez \& Zurita, 2013; Green, Felix, Sharkey, Furlong \& Kras, 2013; Hymel, McClure, Miller, Shumka \& Trach, 2015; Zych, Ortega-Ruiz, Del Rey, 2015; Scheithauera, Smith \& Samara, 2016; Smith, 2016). A la vez, es inobjetable que conforme transcurre el tiempo no sólo es mayor el número de disciplinas que se involucran en esta empresa, sino que se prevé que esta tendencia se agudice en el futuro tal como argumentan Henry \& Bracy (2012). Esta tendencia es una expresión de la complejidad de la violencia escolar puesto que solo desde diferentes aproximaciones de conocimiento, se puede adoptar un examen comprehensivo y holístico, que haga aprehensible este fenómeno desde todas las ópticas psicológicas, sociales, culturales políticas, legales, educativas y económicas que lo configuran (Hymel \& Swearer, 2016).

En los tiempos actuales se dispone de una cantidad extraordinaria de estudios en donde colaboran expertos de diferentes disciplinas quienes emplean múltiples técnicas de investigación. De hecho, algunas de ellas son muy innovadoras porque recuperan el uso de las tecnologías de la información y de las redes sociales como espacios donde se ha extendido la convivencia escolar y, también, donde se han generado manifestaciones inéditas de violencia escolar, como el ciberacoso. Sin embargo, nadie dudaría en aseverar que todavía no se conocen con suficiencia y profundidad sus dinámicas y procesos distintivos en las escuelas (Brown y Munn, 2008; Green, Felix, Sharkey, Furlong \& Kras, 2013; Scheithauera. Smith \& Samara, 2016). Preguntarse entonces ¿qué tipo de información se necesita para tomar mejores decisiones?, ¿cómo se puede recabar?, ¿a quién se debe considerar como informante?, ¿qué tipo de temas se deben incluir?, ¿es pertinente utilizar los instrumentos estandarizados en contextos geográficos y culturales diferentes?, entre muchas otras cuestiones, implica inevitablemente realizar un balance de las tendencias recientes alrededor de los marcos conceptuales, teóricos, analíticos y metodológicos utilizados en la investigación de la violencia escolar (Hymel \& Swearer, 2015; Smith, 2016). Una parte de este balance conviene ubicarla en el estudio del papel que distintos miembros de las comunidades escolares tienen en la indagación empírica de este fenómeno, en especial del alumnado debido a que es un grupo invisibilizado y excluido históricamente de la participación en asuntos relevantes de la escuela y el sistema educativo.

Para emprender el análisis del papel del estudiantado mexicano respecto a la investigación empírica sobre la violencia en las escuelas, es necesario exponer varios planteamientos que constituyen puntos de partida en este documento. En años recientes, la indagación empírica de la violencia escolar ha transitado por distintas rutas (Brown \& Munn, 2008; Gómez \& Zurita, 2013; Green, Felix, Sharkey, Furlong \& Kras, 2013; Hymel, McClure, Miller, Shumka \& Trach, 2015; Hymel \& Swearer, 2015; Zych, Ortega-Ruiz, Del Rey, 2015; Scheithauera, Smith \& Samara, 2016; Smith, 2016). En la década de los años noventa cuando comenzaron a examinarse cuestiones acerca de las nuevas dinámicas que empezaban a manifestarse en torno a la disciplina, indisciplina y violencia, prevaleció el uso de técnicas cualitativas concebidas como las fuentes más pertinentes para obtener la información deseada entre ciertos tipos de población, particularmente de los integrantes de las comunidades de centros educativos, a quienes se contactaba para realizar entrevistas, grupos focales, observaciones en aula y escuelas, entre otras 
técnicas (Gómez \& Zurita, 2013). En estas iniciativas se pretendía describir y comprender los fenómenos asociados a la disciplina y la violencia escolar. Por ello, las poblaciones, contextos y/o casos considerados en este tipo de estudios solían ser más reducidos en cantidad; ya que el interés se orientaba a profundizar en los por qué y los cómo. No obstante, conforme se fueron diversificándose las expresiones violentas y adquiriendo mayor complejidad en distintos ambientes educativos y entre poblaciones numerosas, se aplicaron técnicas de investigación cuantitativas, fundamentalmente encuestas, que tuvieron la finalidad de documentar las tendencias de la violencia a nivel estatal, regional, nacional y según determinados niveles educativos para responder los múltiples qué de este problema. En estos esfuerzos, los propósitos giraban en torno a conocer la evolución, hacer comparaciones en tiempo y espacio entre poblaciones similares o distintas, evaluar los resultados y el impacto de las acciones emprendidas, por ejemplo (Gómez \& Zurita, 2013; Zurita, 2013; Hymel \& Swearer, 2015; Smith, 2016).

Con el transcurrir del tiempo, se fue constatando en México y otros países que la violencia estaba configurándose como un problema complejo en nuestras sociedades (Brown \& Munn, 2008), que no se restringía a ningún tipo de escuela, de sistema educativo, de cultura ni de país (Gómez \& Zurita, 2013; Hymel \& Swearer, 2015; Scheithauera. Smith \& Samara, 2016, Smith, 2016). Este consenso se extendió rápidamente entre las comunidades de académicos, estudiosos y autoridades ocupadas en su atención y prevención. Las "confrontaciones" entre las investigaciones de corte cualitativo y las de corte cuantitativo también se desvanecieron en la medida en que fue reconocido, por una parte, que ambos tipos proporcionan información pertinente, relevante y útil; y, por otra, que su combinación sustentada en criterios académicos, aumentaba notoriamente las posibilidades de descripción, explicación y comprensión del problema en cuestión. Fue entonces cuando el debate se trasladó hacia otros derroteros como, por ejemplo, las propuestas de nuevas y renovadas técnicas de investigación para dar cuenta de las implicaciones de la tecnología en las dinámicas y procesos de la convivencia escolar; la necesidad de construir o de adecuar instrumentos que pudiesen recolectar información de las expresiones de la violencia escolar particulares a ciertos contextos y expresiones comunes en países culturalmente diferentes (Monks, Smith, Naylor, Barter, Ireland \& Coyne, 2009; Hymel \& Swearer, 2015; Smith, 2016). En conclusión, la evolución del fenómeno relativo a la violencia escolar y el desarrollo de la indagación empírica, demandaron reflexión e innovación sobre las teorías, las metodologías y las técnicas empleadas para su estudio.

El segundo planteamiento tiene que ver con el visible aumento en los últimos años de investigaciones empíricas acerca de la violencia escolar en México, característica que se ha presentado en otros países (Green, Felix, Sharkey, Furlong \& Kras, 2013; Monks, Smith, Naylor, Barter, Ireland \& Coyne, 2009; Hymel \& Swearer, 2015). Ese incremento podría llevar a pensar que cada vez hay más información de la violencia en los centros educativos. Sin embargo, cuando se revisan a detalle las encuestas nacionales y regionales o las encuestas por tipo de nivel educativo, quedan al descubierto varias de sus limitaciones. Por ejemplo, a pesar de la aplicación de diversas encuestas para conocer la violencia en las escuelas mexicanas, todavía no se cuenta con información que permita la observación, el seguimiento y la comparación del fenómeno en amplios horizontes temporales y espaciales. Sin duda, ello se debe en primer lugar a que sus poblaciones objetivo son diferentes; y, además, a que no todas las encuestas se aproximan de la misma manera a la recolección de información del tema que aquí interesa. De hecho, existe un tipo de encuestas, que se distinguen de otras porque únicamente abordan temas de violencia, indisciplina, agresión, entre otros tópicos, como son: la Encuesta sobre Ambientes Escolares en Primarias y Secundarias Públicas del Distrito Federal, 2015 o, bien, la $1^{a}$ Encuesta Nacional sobre Bullying Homofóbico, 2012. También se encuentran aquí las que tienen dentro de otros temas centrales, cuestiones vinculadas con la violencia, disciplina, indisciplina o agresión. Tal es el caso de la encuesta Disciplina, violencia y consumo de sustancias nocivas a la salud en escuelas primarias y secundarias de México, 2007; o, por ejemplo, las Primera, Segunda y Tercera Encuesta Nacional sobre Exclusión, Intolerancia y Violencia en las Escuelas de Educación Media Superior en México 2007, 2009 y 2013. Finalmente, el tipo de encuestas más común corresponde a las que tienen algunas preguntas alrededor de la violencia escolar, acoso entre pares o 
ciberacoso. Aquí se puede mencionar a la Encuesta Nacional sobre Discriminación en México, 2010 o las distintas Evaluaciones sobre las Condiciones de Enseñanza y Aprendizaje en Preescolar, Primarias, Secundarias y Educación Media Superior que el Instituto Nacional para la Evaluación de la Educación (INEE) hizo entre 2014 y 2018.

Sin embargo, conviene resaltar que la gran mayoría de las encuestas existentes, surgieron con otros propósitos, diferentes a los que se han planteado en la investigación exclusivamente dirigida a la violencia, acoso y convivencia escolar. Al hacer una lectura cuidadosa de las definiciones, las categorías, las metodologías empleadas y las preguntas utilizadas, se observa que la información proveniente de las encuestas que se han generalizado en años recientes, posee debilidades y problemas respecto a la sistematicidad, comparabilidad, validez, representatividad, relevancia y hasta de la propia conceptualización de la violencia en las escuelas. Así, aunque a primera vista parece que hay más datos, una parte importante de ellos no constituye información confiable que permita indicar el estado que guarda este problema social a nivel nacional o estatal, según el nivel educativo, el tipo de sostenimiento de la escuela, el turno o la modalidad del servicio educativo, entre otras variables (Gómez \& Zurita, 2013; Zurita, 2013b). Los resultados son, como se podrá imaginar, la confusión e ignoracia de la violencia escolar dado que se suelen extrapolar datos a escalas mayores que corresponden a casos específicos, que se estigmatizan a los individuos involucrados (acosador-víctima), las escuelas, las culturas y las sociedades donde ocurren (Hymel \&Swearer, 2015; Smith, 2016; Zurita, 2016). Este entorno se vuelve todavía más complejo porque, hoy por hoy, la violencia escolar se ha convertido, por la difusión y abordaje de los medios de comunicación, en un tema superficial y trivial que ha motivado la creación de una industria de dudosa calidad orientada supuestamente a su prevención y erradicación (Brown \& Munn, 2008; IIDH, 2012; Gómez \& Zurita, 2013; Zurita, 2013b; 2016; CEPAL, 2018). De hecho, esta situación, sumada al empleo desafortunado de dicho conocimiento, pueden ser algunas de las principales razones que explican las debilidades de numerosas acciones dirigidas a la prevención, atención y erradicación de esa violencia tanto en México como en otros países (Monks, Smith, Naylor, Barter, Ireland \& Coyne, 2009; Smith, 2016; Zurita, 2013b; 2016). Para resumir, parecería que hay más información disponible por la proliferación de encuestas, evaluaciones, sondeos, estudios que incluyen preguntas sobre ese fenómeno, pero en realidad es mayor lo que se desconoce hoy de la violencia escolar.

El tercer planteamiento está relacionado la pertinencia actual y, en cierto sentido, urgencia de la reflexión de la indagación empírica de la violencia escolar. Está ampliamente sustentado que la investigación académica acerca de este fenómeno un campo de estudio nutrido por la participación de numerosas disciplinas (Henry \& Bracy, 2012) que, como en otras tareas del quehacer académico, se abocan a la producción de conocimiento conforme ciertos estándares científicos (Brown \& Munn, 2008, Gómez \& Zurita, 2013). Por una parte, hay un conjunto de datos derivados del control de la disciplina y conducta del alumnado que son construidos por las escuelas y los sistemas educativos como estadísticas institucionales de actos de indisciplina, agresión, acoso y violencia. Por otra parte, hay una gama amplia de actores de la academia, agencias gubernamentales, organismos civiles y organizaciones internacionales que recaban y construyen información numerosa en cantidad y heterogénea en calidad, mediante la aplicación de numerosas técnicas de investigación cuya variación, como se sabe, depende de marcos conceptuales, los diseños metodológicos, las técnicas empleadas en los estudios, así como de la disponibilidad de los recursos necesarios para la consecución de los objetivos planteados. No obstante, lo que hoy requiere someterse a debate es la confiabilidad y validez de los datos existentes, de quién da esa información y de los referentes teóricos y analíticos utilizados para su recolección y examen (Gómez \& Zurita, 2013, Green, Felix, Sharkey, Furlong \& Kras, 2013, Scheithauera. Smith \& Samara, 2016; Smith 2007, Zurita, 2016). Es aquí donde se enfrenta un cúmulo de desafíos que afectan tanto los cursos estratégicos de acción derivados de las decisiones tomadas en materia de políticas públicas, legislación, programas de prevención hasta de los que investigan la violencia escolar y, sin duda, de quienes la enfrentan y atienden día a día en las escuelas. De este modo, la evolución del conocimiento producido, requiere una revisión detallada y cuidadosa de la pertinencia de su utilización en la toma de decisiones correspondientes. 
Estos planteamientos brevemente expuestos arriba, ayudan a delinear un escenario donde se ha configurado un efecto que sin ser intencional puede ser, en principio, alentador en México como en otros países que históricamente se han distinguido por sus deficientes o mínimos esquemas de participación de las y los alumnos de distintos niveles educativos (Fielding, 2007, 2011; Lundy, 2007; Mitra \& Serriere, 2012; Mockler \& Groundwater-Smith, 2015; Zurita, 2016). Se trata del proceso de legitimación de las voces del estudiatado que ha provocado la indagación empírica de la violencia escolar, el cual parecería indicar un primer paso hacia el reconocimiento de su intervención en asuntos que les afectan día a día. Este fenómeno conviene estudiarlo pues cada vez es más paradójico que, aunque las y los estudiantes han sido los destinatarios principales de la gran mayoría de las acciones de política educativa, a lo largo de la historia han sido quienes menos oportunidades han tenido para expresar sus voces y todavía menos las posibilidades reales para que fuesen tomadas en cuenta en la formulación de políticas educativas e, inclusive, en cuestiones que enfrentan a diario en las aulas y escuelas (Zurita, 2016). La participación de NNyA en los procesos investigativos emprendidos para generar propuestas, tomar decisiones, emprender las acciones en asuntos de vital relevancia -como la violencia escolar- y para practicar la educación para la vida democrática en las escuelas, ha motivado a especialistas en el mundo a discutir este fenómeno desde la propuesta Voces del alumnado. A continuación, se presentan algunos planteamientos de esta propuesta que permitirán analizar sobre el papel del estudiantado en la indagación empírica de la violencia en las escuelas mexicanas.

\section{Las voces de las y los alumnos.}

Las Voces de las y los alumnos es un movimiento y una corriente que estudia las formas en que NNyA participan fundamentalmente en los espacios escolares (Tisdall, Kay \& Davis, 2004; Noyes, 2005; MackBeth, 2006; Fielding, 2007, 2011; Lundy, 2007; Mitra \& Serriere, 2012; Mockler \& Groundwater-Smith, 2015). Como se puede suponer, la presencia de este movimiento ha sido mayor a partir de la Convención de los Derechos del Niño de 1989; pero tiene importantes antecedentes en la obra de diversos pensadores clásicos, John Dewy, Paulo Freire, entre otros. Las Voces está vinculada estrechamente con modelos de democracia participativa y con movimientos a favor de los derechos de NNyA. La atención prioritaria de esta corriente está puesta en la escuela porque esta institución tiene la encomienda histórica de la enseñanza y ejercicio cotidiano de los principios democráticos en los primeros años de vida, cuyo comportamiento cívico y la conformación una identidad política se desplegará en la sociedad una vez que sean reconocidos como ciudadados (Tisdall, Kay \& Davis, 2004; Noyes, 2005; MackBeth, 2006; Fielding, 2007, 2011; Lundy, 2007; Mitra \& Serriere, 2012; Mockler \& Groundwater-Smith, 2015).

Como en otros desarrollos teóricos, la discusión acerca de esta perspectiva ha sido intensa y ha abarcado desde la pertinencia del uso del término Voces hasta la multiplicidad de procesos participativos que la incluyen. A continuación, se expondrán algunos de los aspectos que han generado cierta controversia. En primer lugar, para algunos críticos, la persistencia de concepciones y discursos donde el alumnado es concebido de manera pasiva, subordinada e incompetente puede ser, de algún modo, reafirmada con el término Voces pues la gran mayoría de las indagaciones empíricas las toman en cuenta como una fuente de información de diversos tópicos escolares y/o educativos. Sin embargo, los miembros de las Voces destacan que esa utilización obedece a la búsqueda de legitimación de las propuestas formuladas y emprendidas por los docentes, directivos y otras autoridades. Al hacerlo, señalan que la aplicación de diversas técnicas para recabar la información entre el estudiantado -tales como entrevistas, historias de vida, grupos focales, sondeos, encuestas e, incluso, consultas con estos grupos poblacionales-; por sí mismas no constituyen ningún acto facultativo de NNyA como productores de conocimiento ni tampoco los configura como actores autónomos y con agencia en concordancia con los principios de una democracia participativa. 
Adicionalmente, algunos críticos de este movimiento sostienen que la fuerza inherente que parece acompañar a la noción Voces, se desvanece inmediatamente si no hay quién las escuche y si no se precisa puntualmente cuándo, cómo, en dónde, por qué y para qué habrían de ser oídas. Según sus críticos, si no se toman en cuenta esos elementos, las Voces se erige como un movimiento ingenuo que no aborda las relaciones de poder que estructuran los procesos de enseñanza y aprendizaje en el ámbito escolar. No obstante, sus promotores coinciden en señalar que el análisis de esos aspectos en los instrumentos que rigen la gestión y la convivencia escolar reflejan a cabalidad las tareas que están todavía pendientes en lo concerniente al impulso de la participación de NNyA. Además, la participación debe estar en sintonía con el diseño institucional, organizacional y normativo de las escuelas como en la formulación de políticas y programas orientados a estas poblaciones (Zurita, 2013b, 2016).

Otro asunto que ha provocado cuestionamientos adicionales al término Voces está relacionado con el desdibujamiento de la desigualdad, inequidad y exclusión que pueden experimentar NNyA de múltiples formas en las escuelas como en otros espacios. La noción de Voces parecería que no reconoce diferencias relevantes entre las y los alumnos que las expresan. Pero, los especialista de este movimiento han señalado que la investigación de las Voces considera variables tales como sexo, edad, religión, nivel socioeconómico, lugar de residencia, condición migratoria, pertenencia a grupos étnicos, trayectoria escolar, nivel educativo, tipo de escuela, entre otras. De hecho, si no se tomasen en cuenta variables como ésas, la aplicación de esta propuesta no podría ir más allá de la descripción del fenómeno empírico en un contexto determinado (Mitra \& Serriere, 2012; Mockler \& Groundwater-Smith, 2015). En otras palabras, la perspectiva de las Voces abre grandes posibilidades para comprender y explicar los avances y desafíos de la participación de NNyA en las escuelas de nuestros tiempos mostrando las deudas pendientes respecto a la inclusión, equidad, respeto y ejercicio de los derechos humanos de estos grupos.

Una última cuestión que subyace a esta propuesta, es la crítica que hacen respecto a que quienes participan, en este caso NNyA, deben tener tanto la autonomía como la identidad que les permita reconocerse como titulares de derechos y ser reconocidos por los otros como actores sociales con agencia (MacBeth, 2006; Fielding, 2007, 2011; Lundy, 2007). Ahora bien, esa autonomía e identidad no sólo requiere ser garantizada mediante diversos instrumentos legales de diferente status jurídico, o, bien, con la existencia de esquemas formales o institucionales para participar (Zurita, 2016; 2018). Se requiere que en todos los ámbitos donde se desenvuelve la vida cotidiana de estas poblaciones, disfruten del reconocimiento de sus derechos e identidades y que dispongan de espacios propicios para el ejercicio efectivo de su derecho a la participación.

En congruencia con el objetivo principal de este documento, conviene subraya que algunos especialistas de las Voces, como Michael Fielding $(2007,2011)$ se han enfocado en el análisis de las interacciones que se configuran a propósito del aprendizaje y la enseñanza entre NNyA y aquellos adultos que constituyen el equipo docente y directivo de las escuelas. Esta interacción comprende desde aquellos contactos más básicos para la obtención de información donde NNyA tienen una participación pasiva y subordinada porque responden a lo que los otros quieren saber, cómo lo quieren conocer y cuándo lo quieren indagar; hasta aquellos donde quienes proponen los temas, construyen las propuestas para recolectar información, toman las decisiones y emprenden las acciones que de ellas se desprendan, son NNyA, además de los adultos, puesto que la participación no está limitada a la edad o a la función que cumplen en la escuela. Esto es posible en entornos donde, en principio, este último tipo de intervención es legal, legítima y se despliega dentro del modelo de democracia participativa en la escuela. Con esta propuesta, Fielding llama la atención hacia la posibilidad de construir en las sociedades contemporáneas, procesos más dinámicos y complejos de esa interacción que conduzcan a transformar los modos convencionales en que históricamente han sido pensados NNyA en las instituciones escolares.

Ahora bien, para profundizar en el análisis, Fielding construyó un modelo de los patrones de asociación (Patterns of partnership) que contiene seis tipos de interacción alrededor de las cuales se advierten diferentes concepciones del alumnado como: a) fuentes de información para 
conocer su bienestar y progreso; b) participantes activos en el diálogo y discusión que son actividades propuestas y coordinadas por los adultos (docentes, directores, entre otras figuras de las comunidades escolares); c) co-investigadores bajo la tutela del equipo escolar con alto perfil y apoyo activo estudiantil; d) creadores de conocimiento en donde las y los alumnos asumen un papel más relevante en la conducción pero cuentan con el apoyo del equipo docente y directivo escolar; e) autores en el mismo nivel los docentes y directivos de las escuelas pero sin autonomía total ni plena autoridad para plantear temas, hacer propuestas, tomar decisiones; $y$, finalmente, $f$ ) figuras centrales de procesos de aprendizaje intergeneracional correspondientes a modelos de democracia participativa, donde la responsabilidad y el compromiso son compartidos por todos los que colaboran y entre los cuales no hay ningún tipo de diferencia o jerarquía (Fielding, 2011, p. 67).

Aunado a ello, habría que añadir que la singularidad de la propuesta de Fielding es que, en esta interacción, NNyA son concebidos de diferentes maneras de acuerdo con su participación en la producción de conocimiento y según los principios de legalidad y legitimidad que la respalden. Sin embargo, este abanico de opciones no representa un modelo evolutivo ni en el tiempo ni en los tópicos en torno de los cuales se construye conocimiento. Por eso, la utilidad del modelo de Fielding es inobjetable ya que, por ejemplo, se podría analizar de qué forma interactúan el estudiantado y el equipo docente y directivo, a lo largo de un período, en un espacio delimitado y por un tema concreto. Como también se podría conocer cómo son las interacciones entre NNyA y los adultos en un momento y entorno, pero en función de distintos asuntos escolares y educativos.

Reconociendo entonces la pertinencia del modelo de Fielding, la configuración de las NNyA como productores de conocimiento respecto a la violencia escolar, cobra características distintivas porque el discurso hegemónico está basado en su conceptualización "estrecha" y "superficial" que identifica a las y los alumnos como sus principales protagonistas, especialmente en lo concerniente al acoso entre pares y al ciberacoso (Gómez y Zurita, 2013; Hymel \& Swearer, 2015; Smith, 2916; Zurita, 2016). Si NNyA tienen un papel relevante en la emergencia y, sobre todo, la acentuación de la violencia escolar, se podría preguntar cuáles son las razones por las cuales el estudiantado no asume y no se le acepta un papel más protagónico en la construcción de conocimiento que pueda ser utilizado para la toma de decisiones conducentes a la prevención, atención y eventualmente reducción de este problema. Se trata, según Fielding, de transitar hacia concepciones más activas, propositivas donde el alumnado desarrolle todas sus potencialidades que no es sino reconocerse y ser reconocidos como actores sociales con agencia y como sujetos productores de conocimiento. Estos cambios son apremiantes, ya que para las Voces, las opiniones, propuestas y acciones de NNyA son útiles, pertinentes y relevantes para disfrutar mejores ambientes para la enseñanza, el aprendizaje, la convivencia y, en tanto procesos participativos, son una vía para ejercitar la educación democrática en las escuelas (Fielding, 2007, 2011; Lundy, 2007; Mitra \& Serriere, 2012; Mockler \& Groundwater-Smith, 2015). Por estas razones, las Voces y, en particular, el modelo de Fielding (2011), resulta una herramienta pertinente y relevante para examinar el proceso de legitimación de las voces del alumnado a propósito de la indagación empírica de la violencia en las escuelas mexicanas. Precisamente en el siguiente apartado se presenta este análisis.

La recuperación de las voces del alumnado en la indagación empírica sobre la violencia escolar en México.

$\mathrm{Al}$ echar un rápido vistazo a las investigaciones sobre la violencia escolar, se puede advertir que la indagación empírica ha producido diversas consecuencias. Algunas de ellas tienen que ver con las formas en que NNyA convencionalmente han sido tomados en cuenta en los análisis como población objetivo en el diseño y aplicación de técnicas cualitativas, tales como entrevistas, observación participante, historias de vida como parte de estudios micro; o a partir de la mitad de los años 2000, como población entrevistada cuando comenzó el boom de encuestas para recabar información de las experiencias, opiniones y valoraciones en torno de la violencia escolar y, en 
especial, del acoso escolar entre pares. En tiempos más recientes, el alumnado continuó estando presente como población objetivo cuando se propagaron estudios basados en la combinación de técnicas cualitativas y cuantitativas para analizar estos fenómenos violentos en las escuelas (Gómez \& Zurita, 2013; Hymel \& Swearer, 2015; Smith, 2016).

Las preguntas que hoy llaman la atención de los interesados en la violencia escolar, tienen que ver con cuáles son los métodos y las técnicas más eficientes para recolectar la información deseada, las más robustas de acuerdo con los estándares científicos establecidos, las más pertinentes para prevenir los actos no deseados, las más económicas para la diversidad de instancias gubernamentales y no gubernamentales involucradas en las iniciativas así como por los cuantiosos recursos necesarios para su inversión en las tareas de prevención, atención y eliminación de este problema social (Brown \& Munn, 2008). De forma inexplicable, el papel de NNyA estudiantes en el marco de las investigaciones no ha sido sometido a una reflexión profunda a pesar de que con el paso del tiempo se ha confirmado su consideración obligada en cualquier indagación empírica en la materia (Hymel \& Swearer, 2015; Smith, 2016); a la vez que persiste el rechazo de la concepción de NNyA como constructores de conocimiento.

La investigación realizada en otros países ha identificado los altos costos negativos para los sistemas educativos contemporáneos que implica que NNyA continúen siendo concebidos como integrantes pasivos, sumisos y subordinados a las decisiones tomadas por otros (Lundy, 2007; Tisdall, Kay \& Davis, 2004; Noyes, 2005; MackBeth, 2006; Fielding, 2007, 2011; Lundy, 2007; Mitra \& Serriere, 2012; Mockler \& Groundwater-Smith, 2015). Este hallazgo conduce a pensar que ahí se encuentra un factor explicativo de los escasos resultados de las múltiples acciones emprendidas para prevenir la violencia en las escuelas (Gómez y Zurita, 2013; Zurita, 2013b, 2016). Entonces la cuestión central es qué hacer para impulsar los estudios que reconozcan a NNyA como actores con agencia y productores de conocimiento; el cual podría ser recolectado mediante el diseño y aplicación de instrumentos apropiados, como metodologías de investigaciónacción y de técnicas como la voz fotográfica (photo voice) para prevenir la violencia escolar.

Impulsar estos cambios conlleva a observar los diferentes fenómenos alrededor de la violencia escolar desde otra posición que los adultos, ya sean los docentes, directores o padres de familia y autoridades educativas, suelen ignorar o despreciar. Valga como ejemplo, la siguiente experiencia que se tuvo en una investigación colectiva e interinstitucional sobre la violencia en las escuelas primarias y secundarias públicas de la Ciudad de México que se realizó entre 2013 y 2016. Este proyecto estuvo a cargo del Seminario de Investigación en Juventud (SIJ), de la Universidad Nacional Autónoma de México (UNAM) y fue coordinada por José Antonio Pérez Islas. El principal propósito fue ahondar en las condiciones, tanto estructurales como subjetivas, que propician y reproducen ese problema social (Brown \& Munn, 2008). Para ello se diseñaron y aplicaron diversas técnicas cualitativas y cuantitativas (Pérez Islas \& Lara, 2016; Zurita, 2016). En dicha iniciativa, algunos de los expertos participantes utilizaron dentro de su marco conceptual y analítico, planeamientos provenientes de las Voces del alumnado (Zurita, 2016). Dentro de esta investigación, se advirtieron algunos aspectos que vale la pena ahora señalarlos para reflexionar los avances y desafíos actuales en esta materia. Entre los aspectos que más llamaron la atención del equipo participante, resalta la enorme distancia que hay entre, por una parte, el discurso predominante en torno a la violencia escolar que, a su vez, se refleja a su vez en las acciones para su prevención, atención y eliminación; y, por otra parte, las experiencias violentas reales que viven NNyA junto con otros miembros de las comunidades escolares. Esto se percató cuando se les solicitó a NNyA que indicaran su noción de violencia escolar. De manera inmediata y con gran facilidad, proporcionaron la definición prácticamente literal que corresponde a la literatura clásica del acoso escolar entre pares, es decir, a la noción propuesta por Olweus a principios de los años setenta. Como se sabe, en términos generales, ésta consiste en una forma de agresión interpersonal deliberada y repetida en el tiempo que se realiza para provocar algún daño físico, mental o social a una o varias personas y cuya relación está caracterizada por un desequilibrio de poder. Sin embargo, en sus narrativas esa concepción no fue asumida ni como un problema por NNyA ni como un fenómeno que afectara su convivencia escolar. Esto condujo al equipo de 
investigación a pedirles que proporcionaran expresiones puntuales de la violencia escolar que, según su experiencia, afectaban negativamente la convivencia en las aulas y escuelas. Al hacerlo, los tópicos más importantes en opinión de NNyA giraban alrededor de su interacción con los adultos (léase: docentes, directores, supervisores y padres de familia) y con las consecuencias de los conflictos gestados entre éstos, en donde el alumnado se ve de algún modo u otro involucrado. En esos conflictos, las habilidades que tienen NNyA para neutralizar, sobrellevar y remontar dichas consecuencias son distintas de las que tienen para solucionar problemas que tienen con otros estudiantes de los mismos grupos a los que pertenecen, de otros salones o de otros grados de la escuela.

Sin duda, el tipo de conflictos, los modos en que se conceptualizan, las maneras en que se solucionan, los integrantes de las comunidades escolares que interfieren, las normas e instrumentos legales que se invocan y los que se aplican, entre otras variantes dependen de los dispositivos y culturas escolares predominantes que se conjugan con los elementos formalmente establecidos relativos a la gestión escolar (Zurita, 2016). Así, los resultados de esta investigación mostraron caras poco conocidas y documentadas por los estudios recientes que se concentran sólo en el acoso entre pares y dónde las NNyA solo son las fuentes de información de lo que les interesa a los adultos indagar (Pérez Islas \& Lara, 2016; Zurita, 2016).

Ahora bien, al recuperar las opiniones de las NNyA se advirtieron que efectivamente sus voces varían de acuerdo con su sexo, edad, experiencia escolar, trayectoria escolar, nivel socioeconómico, tal como Fielding y otros autores de las Voces lo han indicado. Pero ello no impidió dar cuenta que debajo de los discursos que se han vuelto hegemónicos en las escuelas mexicanas en torno a la prevención, atención y eliminación de la violencia, persisten interacciones interpersonales que, alimentadas por los dispositivos institucionales, las culturas escolares y los diseños organizacionales, conforman diversas expresiones violentas en las escuelas; en las cuales NNyA no son los responsables de la propagación de dicho problema social (Pérez Islas \& Lara, 2016; Zurita, 2016).

Fue notorio observar que a pesar de que existen múltiples acciones emprendidas para prevenir, atender y eliminar la violencia escolar en años recientes en México impulsadas por una multiplicidad de actores de diferente escala, además de producir iniciativas desarticuladas, discontinuas, fragmentadas y contradictorias, han incrementado el problema e, incluso, han generalizado la creencia de que el acoso entre pares y el ciberacoso son las principales y más graves expresiones violentas; mientras que se encubren y se les da un peso menor a otras manifestaciones violentas creadas por el propio sistema educativo y sus escuelas, donde otros miembros de las comunidades escolares son sus protagonistas (Zurita, 2013b; 2016).

$\mathrm{Al}$ hacer un examen de la violencia escolar desde las voces del alumnado, es factible recuperar elementos que permiten entender por qué el proceso de legitimación de esas voces aunque se desplegó, no alcanzó otros niveles de participación en la producción de conocimiento, de acuerdo con el modelo de Fielding (2011). Este fenómeno hasta ahora no ha generado la atención por parte de expertos, líderes sociales y autoridades educativas, aun cuando se ha reproducido de nueva cuenta como un doble discurso que es, paradójicamente, la contradicción de los eternos anhelos democráticos que acompañan los fines de la educación y, por otra parte, los atavismos autoritarios que persisten. Este doble discurso, que ha sido un ingrediente histórico en la deuda en torno a la defensa del derecho a la participación de NNyA y su ejercicio efectivo en las escuelas propician que los principios democráticos y el espíritu de los derechos humanos sean ideales inalcanzables.

Hallazgos como ésos, confirman la urgencia de emprender un debate más detallado y profundo sobre los marcos conceptuales, analíticos, metodológicos y técnicos de los estudios abocados a la violencia en las escuelas mexicanas porque sus implicaciones rebasan por mucho la consecución de los objetivos de los estudios, cualesquiera que éstos sean. Como se vio, en esa indagación se juega también el reconocimiento de las NNyA como actores sociales con agencia 
y como sujetos productores de conocimiento de asuntos que, como la violencia escolar, indudablemente les competen. Si bien se ha legitimado la concepción de los NNyA como fuentes de información en los análisis mexicanos de dicho problema social; la indagación empírica podría incorporar metodologías y técnicas de investigación, especialmente de corte cualitativo, como la investigación-acción, el aprendizaje-servicio o la voz fotográfica, que contribuyan a consolidar la concepción de las NNyA como algo más que fuentes de información cuya utilización permite legitimar las decisiones y las acciones tomadas por otros (Fielding, 2007, 2011; Mitra \& Serriere, 2012; Mockler \& Groundwater-Smith, 2015; Zurita, 2013, 2016).

Como se puede observar, el examen y reflexión emprendidos en este documento son tareas apremiantes cuando la Convención sobre los Derechos del Niño, a la que se adhirió México poco después de su creación, está a punto de celebrar su trigésimo Aniversario (CEPAL, 2018). Además, porque en México aunque se cuentan con importantes instrumentos normativos de diferente estatus jurídico que reconocen la participación de NNyA en diferentes ámbitos como las escuelas y la educación, continúan sin tener la legitimidad para hacerlo (Zurita 2013, 2016, 2018). Esta situación es todavía más grave porque este marco jurídico incluye, por ejemplo, más de treinta leyes estatales que desde mediados del 2000 han sido dirigidas a la prevención, atención y eliminación de la violencia escolar y ahí se estipulan algunas participaciones concretas de NNyA (Zurita, 2013b, 2016, 2018), Adicionalmente, se encuentra la LGDNNA, la cual entre los veinte derechos que establece, se incorporó por primera vez en la historia de los derechos de NNyA, el derecho a la participación (UNICEF, 2018). El artículo 71 establece que NNyA tienen derecho a ser escuchados y tomados en cuenta en los asuntos de su interés, conforme a su edad, desarrollo evolutivo, cognoscitivo y madurez. En el ámbito de sus respectivas competencias, las autoridades de los distintos órdenes de gobierno están obligados a disponer e implementar los mecanismos que garanticen la participación permanente y activa de NNyA en las decisiones que se toman en los ámbitos familiar, escolar, social, comunitario o cualquier otro donde se desenvuelve su vida. Asimismo, NNyA tienen derecho a que las diferentes instancias gubernamentales, les informen cómo su opinión ha sido valorada y tomada en cuenta. Con instrumentos legales como esa Ley, la participación de NNyA en México no puede estar sujeta a la voluntad de docentes, directores o autoridades educativas puesto que es un derecho que debe ser reconocido y ejercido plenamente en las escuelas y en otros espacios donde se desenvuelve su vida. Lamentablemente esta realidad es aún lejana para NNyA mexicanos (Zurita, 2010, 2013b, 2016, 2018). De hecho, todavía se observa tanto en la Convención sobre los Derechos del Niño como en la LGDNNyA la presencia de concepciones tuteladas de la participación de NNyA, cuyo ejercicio pleno se condiciona a la edad, madurez y otros aspectos (Lundy, 2007, Zurita, 2013, 2016, 2018; CEPAL, 2018). Asimismo, en los avances en la creación reciente de instrumentos legales internacionales y nacionales para prevenir la violencia en las escuelas impulsados por actores internacionales poderosos, tal como se refleja en la Resolución aprobada por la Asamblea General de las Naciones Unidas el 19 de diciembre de 2016 que aboga por la protección de los niños en contra del acoso, reconocen que todavía no existen las condiciones para el ejercicio real del derecho a la participación de NNyA en tareas relativas a la prevención, atención y eliminación de dicho problema social.

Por ello, no es casual que tanto los expertos como los representantes de los organismos civiles nacionales e internacionales ocupados en la defensa de los derechos de NNyA consideran que los mayores desafíos mayores se encuentran en el contenido, instrumentalización y aplicación de los instrumentos legales en ámbitos como las escuelas; ya que persiste la idea de que los adultos -ya sean los docentes, directores y, sin duda, los padres de familia-, son los únicos que deben participar y los únicos que deben tomar las decisiones concernientes a la violencia escolar porque saben lo que es mejor para el bienestar y la protección de NNyA. De este modo, los retos que enfrenta el alumnado mexicano para tener un papel activo y propositivo en la indagación empírica de la violencia escolar, terminan siendo problemas de la participación, tal como lo expresa Fielding y otros representantes de la perspectiva de las Voces. En otras palabras, parecería que ser reconocidos como sujetos productores de conocimiento no puede desligarse de su reconocimiento como actores con agencia. Así, NNyA mexicanos no pueden producir conocimiento porque 
enfrentan diferentes obstáculos jurídicos, institucionales, organizacionales y culturales que les impiden participar. Aunque ya se reconoce el derecho a la participación de NNyA en México, la concepción de la participación es una visión tutelada. En términos del sistema educativo, desde décadas atrás se puede observar en diferentes leyes -como la Ley Orgánica de la Educación Pública de 1942, la Ley Federal de Educación de 1973 o la Ley General de Educación vigente-, que se ha tenido una visión condicionada de la participación del alumnado puesto que se ha reconocido que esta población puede emprender ciertas actividades, pero también se les ha prohibido su intervención en asuntos medulares de las escuelas y el sistema educativo. Asimismo, otra limitación está relacionada con la disponibilidad de una sola modalidad institucional de participación que es la Asociación o Sociedad de Alumnos; y que, además, no está reconocida para todos los niveles del sistema educativo. Pero estos problemas son más paradójicos cuando se piensa que la literatura convencional de la violencia escolar y la opinión difundida por los medios de comunicación, muestran a NNyA como los principales causantes de este problema social, pero simultáneamente se les impide que asuman un papel más activo en la producción de conocimiento que podría ser empleado para evitar dicho fenómeno (Brown \& Munn, 2008; McCluskey, Brown, Munn, Lloyd, Hamilton, Sharp \& Mackleod, 2013).

Las Voces del alumnado representa una propuesta de inclusión de estos integrantes de las comunidades escolares que históricamente han sido excluidos de cualquier proceso de toma de decisiones escolares y educativas. Cabe agregar esta inclusión entendida como un proceso, que no pretende sobreponer las voces de NNyA a las de los otros miembros de las comunidades escolares. Por el contrario, se trata de establecer las condiciones para que cualquiera de sus integrantes, sin distinción de ningún tipo, pueda expresar su opinión, compartir su experiencia y participar en las acciones que se decida emprender. En consecuencia, tal como lo señalan los especialistas de la perspectiva de las Voces, se apela al diálogo y la reflexión sustentadas en la mutualidad, reciprocidad y respeto entre $\mathrm{NNyA}$, docentes, directores, padres de familia y otros integrantes de las comunidades para conocer, intercambiar y delinear las distintas perspectivas, experiencias y propuestas que tienen acerca de la violencia escolar. Si bien es un modelo que se puede aplicar de manera diacrónica y sincrónica para profundizar en los avances y desafíos en torno a las Voces del alumnado en un contexto y periodo temporal determinados, en este trabajo se ha optado por reflexionar cuál ha sido su papel en la indagación empírica de la violencia escolar para conocer qué tipo de interacción entre NNyA y el equipo escolar se despliega, de acuerdo con el modelo de Fielding (2011).

Como lo han sostenido Fielding y otros académicos, las voces del estudiantado pueden ser el corazón de las escuelas, ser expresadas a través de múltiples canales institucionales y no institucionales, desplegarse en torno a cuestiones asuntos centrales como la enseñanza y el aprendizaje, estar reflejadas en el ethos del sistema educativo y las escuelas, ser tomadas en cuenta para todas las acciones donde NNyA estén relacionados. Aquí se ha examinado el proceso de legitimación de las voces del alumnado a propósito de la indagación empírica sobre la violencia escolar. Sin embargo, en la medida en que se identificó que su participación en este proceso no va más allá de una interacción donde ese grupo es considerado como una fuente de información; se mostró también que ello se explica porque, aunque hay nuevos instrumentos legales como la LGDNNA que reconoce en México el derecho a la participación de NNyA lo hace desde una visión tutelada. De modo tal que no hay actualmente condiciones en las escuelas y el sistema educativo para que ese grupo sea considerado como sujeto social con agencia ni como sujeto productor de conocimiento de un tema donde NNyA podrían decir y aportar mucho, como es la violencia escolar.

\section{Conclusiones}

En México existe desde 2014, la LGDNNA que, junto con otros instrumentos jurídicos, reconoce el derecho a la participación de NNyA en diferentes ámbitos, como el escolar (Zurita 2013b; 2016, 2018). No obstante, el alumnado encuentra a diario diversos obstáculos formales -como los instrumentos normativos, las modalidades existentes de participación, la organización escolar- e informales -como la cultura escolar, los valores, las identidades, la legitimidad-, cuando busca 
incidir en cuestiones centrales de la vida de las escuelas, como por ejemplo la construcción de normas para la convivencia escolar (Zurita, 2013b, 2016, 2018). En contraste, docentes y directores aprueban la participación de los NNyA en asuntos que no implican un cuestionamiento de las formas en que históricamente han sido excluidos, subordinados e invisibilizados (Zurita, 2018).

Si la atención se coloca en el papel de NNyA en la indagación empírica para formular acciones para prevenir, atender y eliminar la violencia escolar, se manifiestan otras paradojas que interfieren y retrasan la posibilidad de que estos grupos sean considerados de manera activa, propositiva en tanto sujetos sociales con agencia y como productores de conocimiento. Entre éstas sobresalen la carencia de auténticos esquemas o modalidades de participación para estas poblaciones en los centros escolares y en los sistemas educativos; la desconfianza, condicionamiento o rechazo en torno a la participación del alumnado, pero estas posturas se acentúan más conforme disminuye la edad y el nivel educativo; el predominio de la lógica adultocéntricas en escuelas que siguen siendo verticales y jerárquicas.

En el caso especial del tema que aquí interesa, se observa la solicitud u obligación de la participación del estudiantado para proporcionar información exclusivamente de los asuntos que les interesa a las autoridades escolares y educativas, tales como el acoso escolar entre pares o el ciberacoso; en menoscabo de otras expresiones de violencia como las que existen de los docentes $\mathrm{y}$ directores hacia el alumnado, entre el equipo docente y directivo, entre padres de familia y equipo docente, por ejemplo (Gómez y Zurita, 2013; Zurita, 2013b, 2016, 2018). Esa indagación empírica está, además, sustentada en la aplicación de metodologías y técnicas de investigación que no permiten a NNyA tener un papel más activo, participativo y democrático; a diferencia de otras, señaladas antes, que si posibilitan y propician que esos grupos sean quienes identifiquen, desde sus experiencias, miradas y voces, los temas que vale la pena reflexionar.

Un asunto estratégico que tendrá que cambiar además de la eliminación de la visión tutelada del derecho a la participación y su ejercicio en distintos ámbitos como las escuelas y el sistema educativo que derivaría en el diseño e implementación de todas las acciones necesarias para que NNyA sean reconocidos como sujetos sociales con agencia y como sujetos productores de conocimiento. Asimismo, habrá que exigir que las instancias gubernamentales cumplan con las obligaciones adicionales que tienen, según la LGDNNA, relativas al diálogo y devolución de la información con quienes la proporcionaron. Esto supone el diseño y operación de mecanismos de rendición de cuentas y transparencia por parte de las autoridades respecto a cómo se utilizó la información otorgada por las NNyA y qué resultados e impacto tuvo su empleo en las iniciativas desplegadas.

El proceso de legitimación de las voces del estudiantado ha ocurrido hasta ahora en un contexto donde se han gestado cambios relevantes en el terreno de la investigación educativa en México y, sin duda alguna, tiene similitudes en otros países. Por ahora, dos de ellos merecen ser mencionados aquí: el primero es la institucionalización de la investigación de la violencia escolar en el ámbito académico; y el segundo es el despliegue de estudios desde las miradas, experiencias y voces por parte de la multiplicidad de actores escolares -entre los cuales destacan NNyA-, así como también de todos aquellos actores de distinta escala que intervienen en el sistema educativo y sus escuelas mediante políticas, programas, legislación y otras iniciativas (Gómez y Zurita, 2013; Zurita, 2013b, 2016). En este panorama, es incuestionable el fin del predominio de las grandes explicaciones hegemónicas sobre la educación sustentadas en visiones lineales, mecánicas, homogéneas y ahistóricas cuyos actores, a pesar de su relevancia, han sido históricamente invisibilizados, subordinados y menospreciados.

En conclusión, de acuerdo con el modelo de Fielding (2011), la interacción entre NNyA y adultos que se conforma en el contexto de la indagación empírica acerca de la violencia escolar en México, no llegaría más allá del nivel donde el alumnado sólo es considerado como una fuente de información. De este modo, los discursos esgrimidos por los adultos que ocupan posiciones de autoridad y poseen legitimidad entre la comunidad escolar y el sistema educativo, asumen 
abiertamente un papel de guardianes del bienestar, rechazando e, incluso, descalificando cualquier posibilidad de que NNyA pudiesen asumir un papel activo, propositivo y democrático alrededor de un tema que les concierne directamente como es la violencia que se configura en esos espacios. Sin embargo, la recuperación de las voces del alumnado, tal como se expuso en este documento, apela a una transformación mayor ya que alude no sólo al reconocimiento de la titularidad del derecho a la participación por parte de las NNyA y de su pleno ejercicio en las escuelas. Al demandar su reconocimiento como actores sociales con agencia y como sujetos productores de conocimiento, se exige en consecuencia, su inclusión en la formulación de decisiones escolares y educativas, cuestionándose los principios de poder y autoridad que históricamente han moldeado a las instituciones educativas como instituciones jerárquicas, autoritarias y adultocéntricas. En materia de la violencia escolar, la indagación empírica desde la perspectiva de NNyA requiere experimentar cambios profundos; de modo tal que al colocar especial atención para el desarrollo de las enormes potencialidades que estas poblaciones tienen, disfruten de los derechos que la ley establece no sólo para nombrar asuntos diversos vinculados con ese problema social, sino también para que gocen de la legitimidad para participar en las decisiones y acciones que se tomen para prevenirla y eliminarla. Estos son, en conclusión, algunos de los retos que, sin reconocer fronteras de ningún tipo, acompañan la indagación empírica de la violencia en las escuelas mexicanas.

\section{Referencias}

Brown, J. A., \& P. Munn. (2008). School violence' as a social problem: Charting the rise of the problem and the emerging specialist field. International Studies in Sociology of Education, 18(3), 219-230. Doi: https://doi.org/10.1080/09620210802492807

Comisión Económica para América Latina y el Caribe (CEPAL). (2018). América Latina y El Caribe A 30 Años de la Aprobación de la Convención Sobre los Derechos del Niño. Santiago Chile: Naciones Unidas.

Fielding, M. (2007). Beyond "Voice": New roles, relations, and contexts in researching with young people. Discourse: studies in the cultural politics of education, 28(3), 301-310. Doi: https://doi.org/10.1080/01596300701458780

Fielding, M. (2011). Patterns of partnership: Student voice, intergenerational learning and democratic fellowship. En: N. Mockler \& J. Sachs. Rethinking educational practice through reflexive inquiry: Essays in honour of Susan Groundwater-Smith (pp. 61-75). Dordrecht: Springer.

Fondo de las Naciones Unidas para la Infancia (UNICEF). (2018). Los derechos de la infancia y la adolescencia en México. México: Fondo de las Naciones Unidas para la Infancia.

Gómez, A., \& Zurita Rivera, U. (2013). El estudio de la violencia escolar, orígenes y tendencias. En: A. Furlan y T. C. Spitzer S. (Coord. Gral.) Convivencia, disciplina y violencia en las escuelas 2002 - 2011 (pp. 183 - 222). México: COMIE - ANUIES.

Green, J.G., Felix, E.D., Sharkey, J.D., Furlong, M.J., \& Kras, J.E. (2013). Identifying bully victims: Definitional versus behavioral approaches. Psychological Assessment, 25, 651657.

Henry, S., \& Bracy, N. L. (2012). Integrative Theory in Criminology applied to the complex social problem of school violence. A complex problema in need of an interdisciplinary analysis. En: R. Allen, W. H. Newell y R. Szostak (eds). Case Studies in Interdisciplinary Research. (pp. 259-282). Thousand Oaks, CA: Sage.

Hymel, S., McClure, R., Miller, M., Shumka, E., \& Trach, J. (2015). Addressing school bullying: Insights from theories of group processes. Journal of Applied Developmental Psychology, 37, 16-24. Doi: https://doi.org/10.1016/j.appdev.2014.11.008

Hymel, S., \& Swearer, S. M. (2015). Four Decades of Research on School Bullying An Introduction. American Psychologist Association, 70(4), 293-299. Doi: http://dx.doi.org/10.1037/a0038928

Instituto Interamericano de Derechos Humanos (2011). X Informe Interamericano de la educación en derechos humanos: Desarrollo en las políticas de convivencia y seguridad escolar con enfoque de derechos. San José de Costa Rica: IDDH. 
Lundy, L. (2007). 'Voice' Is Not Enough: Conceptualising Article 12 of the United Nations Convention on the Rights of the Child. British Educational Research Journal, 33(6), 927942. Doi: https://doi.org/10.1080/01411920701657033

MacBeth, J. (2006). Finding a voice, finding self. Educational Review, 58(2), 195-207. Doi: https://doi.org/10.1080/00131910600584140

McCluskey, G., Brown, J., Munn, P., Lloyd, G., Hamilton, L., Sharp, S., \& Mackleod, G. (2013). Take more time to actually listen': students' reflections on participation and negotiation in school. British Educational Research Journal, 39(2), 287-301. Doi: https://doi.org/10.1080/01411926.2012.659720

Maunder, R. E., \& Crafter, S. (2018). School bullying from a sociocultural perspective. Aggression and Violent Behavior, 38, 13-20. Doi: https://doi.org/10.1016/j.avb.2017.10.010

Mitra, D. L., \&. Serriere, S. C. (2012). Student Voice in Elementary School Reform: Examining Youth Development in Fifth Graders. American Educational Research Journal, 49(4), 743-774. Doi: https://doi.org/10.3102/0002831212443079

Mockler, N., \& Groundwater-Smith, S. (eds.). (2015). Engaging with Student Voice in Research, Education and Community Beyond Legitimation and Guardianship. Cham Heidelberg New York Dordrecht London: Springer.

Monks, C. P., Smith, P. K., Naylor, P., Barter, Ch, Ireland, J., \& Coyne, I. (2009). Bullying in different contexts: Commonalities, differences and the role of theory. Aggression and Violent Behavior, 14(2), 146-156. Doi: https://doi.org/10.1016/j.avb.2009.01.004

Noyes, A. (2005). Pupil voice: purpose, power and the possibilities for democratic schooling. British Educational Research Journal, 31(4), 533-540. Recuperado de: https://www.jstor.org/stable/30032582?seq=1\#page_scan_tab_contents

Pérez Islas, J. A., \& Lira García, I. (2016). Del acoso al conflicto en la escuela. La construcción social de la violencia escolar. México: UNAM.

Scheithauera, H., Smith, P.K., \& Samara, M. (2016). Cultural Issues in Bullying and Cyberbullying among Children and Adolescents: Methodological Approaches for Comparative Research. International Journal of Developmental Science, 10, 3-8. Recuperado de: https://pdfs.semanticscholar.org/3a19/259dbb523f8f08dd44144058bfcd94445f37.pdf

Smtih, P. K. (2016). Bullying: Definition, Types, Causes, Consequences and Intervention. Social and Personality Psychology Compass, 10(9), 519-532. Doi: https://doi.org/10.1111/spc3.12266

Tisdall, E., Kay, M., \& Davis, J. (2004). Making a Difference? Bringing Children's and Young People's Views into Policy-Making. Children \& Society, 18, 131-142. Doi: https://doi.org/10.1002/chi.816

Zurita Rivera, U. (2013a). Las oportunidades para participar de las y los adolescentes en las escuelas secundarias públicas en México. Revista Interacções, 9(26), 229- 260.

Zurita Rivera, U. (2013b). Políticas, programas, legislación, proyectos y acciones gubernamentales y no gubernamentales. En: A. Furlan y T. C. Spitzer Schwartz (Coord. Gral.) Convivencia, disciplina y violencia en las escuelas 2002 - 2011 (pp. 457 -520). México: COMIE - ANUIES.

Zurita Rivera, U. (2016). No gritar, no correr, no empujar. Las miradas, voces y acciones de los estudiantes y los docentes del DF respecto a las normas. México: UNAM.

Zurita Rivera, U. (2018). La participación de niñas, niños y adolescentes en las escuelas mexicanas hacia nuevos horizontes. En Jorge M. Valladares S. (Coord.) Ciudadana y Educación Cívica. Ser y hacer la democracia, (pp. 149 - 179) Yucatán México: Instituto Electoral y de Participación Ciudadana de Yucatán.

Zych, I., Ortega-Ruiz, R., \& Del Rey, R. (2015). Systematic review of theoretical studies on bullying and cyberbullying: Facts, knowledge, prevention, and intervention. Aggression and Violent Behavior, 23, 1-21. Doi: https://doi.org/10.1016/j.avb.2015.10.001 